\title{
The nucleus-nucleus optical potential derived from a complex Skyrme-type interaction
}

\author{
R. Sartor and Fl. Stancu \\ Institut de Physique, Sart Tilman, B-4000 Liege 1, Belgium
}

(Received 8 May 1981)

\begin{abstract}
We construct a complex energy functional to be used in the calculation of the nucleusnucleus optical potential. The functional is related to a Skyrme-like nucleon-nucleon interaction derived from a finite range complex interaction previously obtained from nuclear matter calculations appropriate to heavy ion collisions. Calculations for ${ }^{16} \mathrm{O}+{ }^{16} \mathrm{O}$ reproduce very well the results obtained from the finite range interaction.
\end{abstract}

$\left[\begin{array}{c}\text { NUCLEAR REACTIONS Heavy ion optical potential from a com- } \\ \text { plex energy functional approach. }\end{array}\right]$

At present, several nuclear matter approach calculations of the imaginary part of the nucleusnucleus potential are available. ${ }^{1-4}$ The complexity of the numerical problems involved limits the applicability of this approach either to very high energies $^{1}$ or to light pairs. ${ }^{2,3}$

The present paper is an attempt to establish the validity of a much simpler method based on a complex energy functional. It can be regarded as an extension to the complex domain of the method used previously ${ }^{5}$ for the calculation of the real part of the nucleus-nucleus potential. Owing to its simplicity it could be applied to any pair of nuclei once the density $\rho$ and the kinetic energy density $\tau$ are known.

The basic idea is that the complex effective nucleon-nucleon interaction to be used in the calculation of the optical potential can be parametrized in a way similar to that proposed by Skyrme $^{6}$ for a real interaction. The structure of such a force allows one to express the expectation value of the total energy of a system described by a Slater determinant as a functional of the system densities $\rho$ and $\tau$. This simplifies the application to the nucleusnucleus optical potential, which is calculated as the difference between the total energy at a finite distance $R$ and at infinity:

$$
V(R, k)=E(R, k)-E(\infty, k),
$$

where $k$ is the relative momentum per nucleon. By this we mean that the momentum of nucleus $A_{1}$ is equal to $A_{1} \overrightarrow{\mathrm{k}}$ while that of nucleus $A_{2}$ is equal to
$-A_{2} \overrightarrow{\mathrm{k}}$.

The use of a complex energy functional derived from a Skyrme-type interaction relies on the following observations.

(i) For the real part of the optical potential there is a close quantitative agreement between the energy functional approach results based on the Skyrme interaction ${ }^{5}$ and those obtained from the finite range complex effective interaction ${ }^{3,4}$ derived from the nuclear matter $G$ matrix associated with a deformed Fermi sea, such as, e.g., that represented in Fig. 1

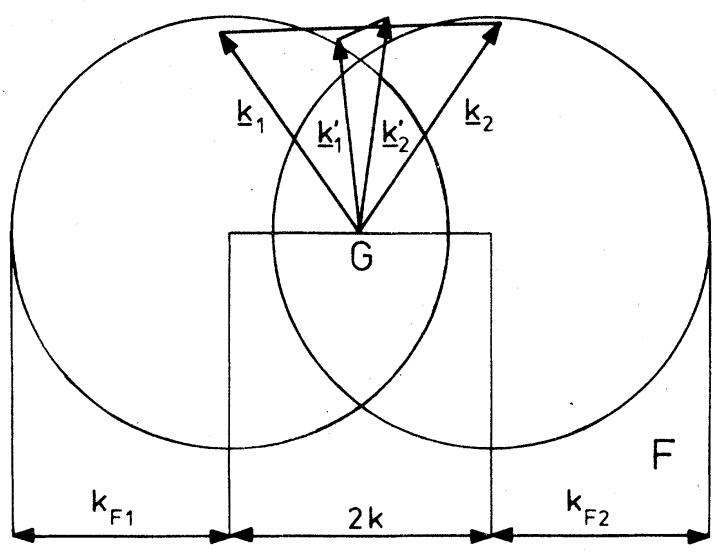

FIG. 1. A typical deformed Fermi sea $F$. Owing to the deformation of $F$, two particles initially inside $F$ can be knocked out of it during an energy conserving collision $\left(\overrightarrow{\mathrm{k}}_{1} \overrightarrow{\mathrm{k}}_{2} \rightarrow \overrightarrow{\mathrm{k}}_{1}^{\prime} \overrightarrow{\mathrm{k}}_{2}^{\prime}\right)$ as shown. For details, see Ref. 2 . 
(ii) The ratio $\xi$ between the real and imaginary parts of the finite range effective force is roughly constant for internucleon distances $r$ up to $3 \mathrm{fm}$. Two typical examples are shown in Fig. 2. The curves have a plateau of almost constant height with respect to $r$ but which varies with $\rho$ and $\tau$. In our examples there is an order of magnitude between the curves (a) and (b) at $r<3 \mathrm{fm}$.

In keeping with remark (ii), we proceed in the following way. We define an $r$-independent $\xi$ as the value taken by the ratio

$$
\xi(\rho, \tau)=\frac{\operatorname{Im} v_{\mathrm{eff}}(r=1 \mathrm{fm})}{\operatorname{Re} v_{\mathrm{eff}}(r=1 \mathrm{fm})},
$$

where $v_{\text {eff }}(r)$ is a spin $S$ and isospin $T$ independent effective interaction obtained by averaging the interaction $v^{S T}(r)$ of Ref. 4 . The reason for choosing $r=1 \mathrm{fm}$ is that both the real and the imaginary parts of $v_{\text {eff }}$ have a minimum around $1 \mathrm{fm}$.

The average over $S$ and $T$ is made according to the formula

$$
v_{\mathrm{eff}}(r)=\frac{\sum_{S T}(2 S+1)(2 T+1) W^{S T}(r) v^{S T(r)}}{\sum_{S T}(2 S+1)(2 T+1) W^{S T}(r)},
$$

with $W^{S T}(r)$ given by

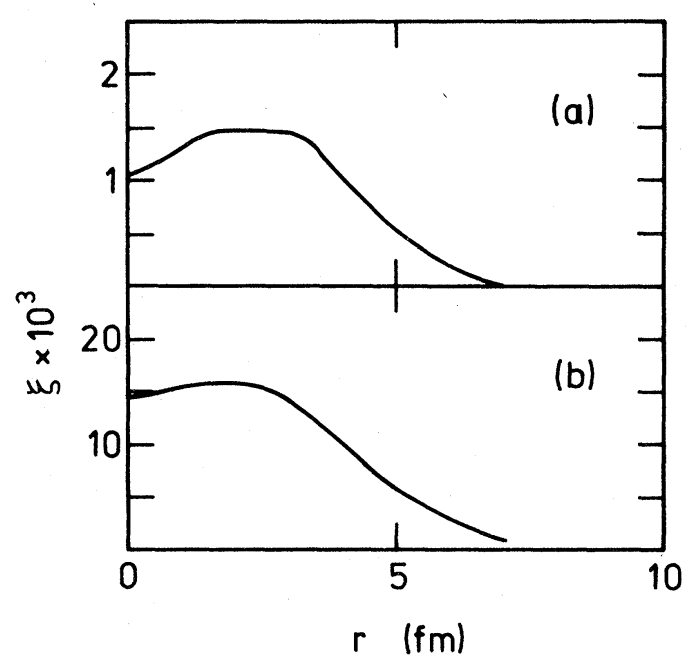

FIG. 2. The ratio $\xi=\operatorname{Im} v_{\text {eff }}(r) / \operatorname{Re} v_{\text {eff }}(r)$ as a function of the internucleon distance $r$ for two different deformed Fermi seas: (a) $k_{F_{1}}=1.63890, k_{F_{2}}=1.54759, k=0.25$ $\left(\mathrm{fm}^{-1}\right)$ or equivalently $\rho / \rho_{0}=2, \tau^{(2)}=0.60414 \mathrm{fm}^{-5}$; (b) $k_{F_{1}}=0.98073, k_{F_{2}}=0.77426, k=0.5\left(\mathrm{fm}^{-1}\right)$ or $\rho / \rho_{0}=0.5, \tau^{(2)}=0.06655 \mathrm{fm}^{-5} \cdot \rho_{0}$ is the normal nuclear matter density.

$$
\begin{aligned}
& W^{S T}(r)=\sum_{L}(2 L+1) \int_{F} d \overrightarrow{\mathrm{k}}_{1} d \overrightarrow{\mathrm{k}}_{2} j_{L}{ }^{2}\left(k_{12} r\right) \\
& (L+S+T \text { odd })
\end{aligned}
$$

In Eq. (4), the momentum integrations are carried over a Fermi sea $F$, such as the one depicted in Fig. 1; the relative momentum $\overrightarrow{\mathrm{k}}_{12}$ is defined as

$$
\overrightarrow{\mathrm{k}}_{12}=\frac{1}{2}\left(\overrightarrow{\mathrm{k}}_{1}-\overrightarrow{\mathrm{k}}_{2}\right)
$$

while $j_{L}$ denotes the spherical Bessel function of order $L$. This averaging procedure insures that $v_{\text {eff }}(r)$ reproduces the complex total energy of the deformed Fermi sea in the Born approximation. We refer to Refs. 4 and 7 for further details.

In Ref. 4, calculations have been made for a series of deformed Fermi seas. At given density $\rho$ and relative momentum per nucleon $k$, this series consists of a succession of configurations of constant volume formed of overlapping spheres with centers separated by $2 k$ and radii $k_{F_{1}}$ and $k_{F_{2}}$. The example given in Fig. 1 is a particular case where $k_{F_{1}}=k_{F_{2}}$ and it corresponds to a maximum in the kinetic energy density $\tau$, or alternatively in the intrinsic kinetic energy density $\tau^{(2)}$ defined as

$$
\tau^{(2)}=\tau-\frac{j^{2}}{\rho},
$$

where $j$ is the current density. At given $\rho$ and $k$, the minimum of $\tau$ is obtained for a single sphere with a radius such that the volume is conserved. Therefore, $\tau_{\min }^{(2)}$ is given by

$$
\tau_{\min }^{(2)}=\frac{3}{5}\left(\frac{3 \pi^{2}}{2}\right)^{2 / 3} \rho^{5 / 3} \text {. }
$$

At given $k$, the values of $\rho$ and $\tau^{(2)}$ determine uniquely $k_{F_{1}}$ and $k_{F_{2}}$. We have extracted the ratio $\xi$ for the series of $\left(k, \rho, \tau^{(2)}\right)$ values considered in Ref. 4. For other values necessary in the calculation, the ratio $\xi$ has been obtained by a Lagrange interpolation. For fixed $k$ and $\rho, \xi$ is shown as a function of $\tau^{(2)}$ in Fig. 3.

Following remark (i), we replace $\operatorname{Re} v_{\text {eff }}$ with the Skyrme interaction $v_{\text {SK }}$ described in Ref. 8. Then the complex interaction we deal with has the form

$$
v_{\mathrm{SK}}^{(c)}(\overrightarrow{\mathrm{r}})=\left\{1+i \xi\left[\rho, \tau^{(2)}\right]\right\} v_{\mathrm{SK}}(\overrightarrow{\mathrm{r}})
$$

where $\overrightarrow{\mathrm{r}}=\overrightarrow{\mathrm{r}}_{1}-\overrightarrow{\mathrm{r}}_{2}$ is the internucleon distance and $\rho$ and $\tau^{(2)}$ are evaluated locally at $\left(\vec{r}_{1}+\vec{r}_{2}\right) / 2$ for a given separation distance $R$ and relative momentum $k$ of the colliding nuclei.

Assumptions such as Eq. (8) have previously been made in simpler forms. In Ref. 9, the 
strength of both the real and imaginary parts were treated as adjustable constants within a double folding procedure. In Ref. 10, also based on an energy functional approach, the derivation of $\xi$ was purely phenomenological. Therefore, one could not have a control on the effect of the Pauli principle, which is of crucial importance for the imaginary part or alternatively for the average nucleon- nucleon cross section relevant to heavy ion collisions. ${ }^{11-13}$ Also, the relative motion was not incorporated in the calculations and $\xi$ was independent of the kinetic energy density.

The interaction (8) gives rise to a complex potential energy density $H_{\text {pot }}^{(c)}$, which is obtained in the same way ${ }^{8}$ as the real potential energy density $H_{\text {pot }}$ of the Skyrme interaction and is related to it by

$$
H_{\mathrm{pot}}^{(c)}\left[\rho(\overrightarrow{\mathrm{r}}), \tau^{(2)}(\overrightarrow{\mathrm{r}})\right]=\left\{1+\xi\left[\rho(\overrightarrow{\mathrm{r}}), \tau^{(2)}(\overrightarrow{\mathrm{r}})\right]\right\} H_{\mathrm{pot}}\left[\rho(\overrightarrow{\mathrm{r}}), \tau^{(2)}(\overrightarrow{\mathrm{r}})\right] .
$$

For $N=Z, H_{\text {pot }}$ has the form

$$
H_{\mathrm{pot}}\left[\rho(\overrightarrow{\mathrm{r}}), \tau^{(2)}(\overrightarrow{\mathrm{r}})\right]=\frac{3}{8} t_{0} \rho^{2}+\frac{1}{16} t_{3} \rho^{3}+\frac{1}{16}\left(3 t_{1}+5 t_{2}\right) \rho \tau^{(2)}+\frac{1}{64}\left(9 t_{1}-5 t_{2}\right)(\vec{\nabla} \rho)^{2},
$$

where $t_{0}, t_{1}, t_{2}$, and $t_{3}$ are the Skyrme interaction parameters. The term proportional to $\rho \tau^{(2)}$ reflects the invariance with respect to a Galilean transformation. ${ }^{14}$ In static calculations, ${ }^{8} j$ vanishes and $\tau^{(2)} \equiv \tau$.

As in Refs. 3 and 5, the Fliessbach two-center model $^{15}$ was used to describe the ground state wave function of the colliding system. The local densities $\rho(\vec{r})$ and $\tau(\vec{r})$ were calculated exactly by using the formulas given in Ref. 16 while the local current density $j(\overrightarrow{\mathrm{r}})$ was approximated by

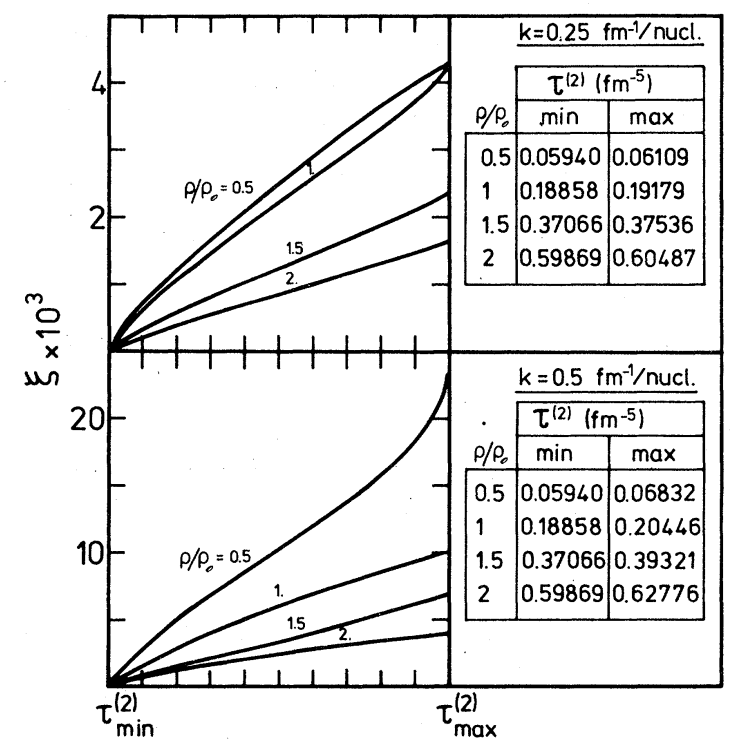

FIG. 3. The $r$-independent $\xi$ [see Eq. (2)] for various densities as a function of the intrinsic kinetic energy density $\tau^{(2)}$ at two values of the relative momentum per nucleon $k$. The $\tau^{(2)}$ scale is linear and divided in units of $\left[\tau_{\max }^{(2)}-\tau_{\min }^{(2)}\right] / 10$, where $\tau_{\max }^{(2)}$ and $\tau_{\min }^{(2)}$ are given in the joint tables for each $\rho / \rho_{0}$. The meaning of $\tau_{\max }^{(2)}$ and $\tau_{\min }^{(2)}$ is explained in the text.

$$
\vec{j}(\vec{r})=\vec{k}\left[\rho_{1}(\vec{r})-\rho_{2}(\vec{r})\right],
$$

where $\rho_{1}(\vec{r})$ and $\rho_{2}(\vec{r})$ refer to the densities at point $\overrightarrow{\mathbf{r}}$ in nuclei 1 and 2 , respectively. In the present calculations, $\overrightarrow{\mathrm{k}}$ is parallel to an axis passing through the nuclear centers. Equation (11) is correct at large $R$, where the antisymmetrization effects in the two-center model are not important. We found that these effects are quite small at smaller $R$ and can therefore be neglected. The ratio $\xi$ also has a local value via $\rho(\vec{r})$ and $\tau^{(2)}(\vec{r})$, which was computed by the Lagrange interpolation mentioned above.

The potential part $E_{\text {pot }}(R, k)$ of the expectation value $E(R, k)$ [see Eq. (1)] is then defined as

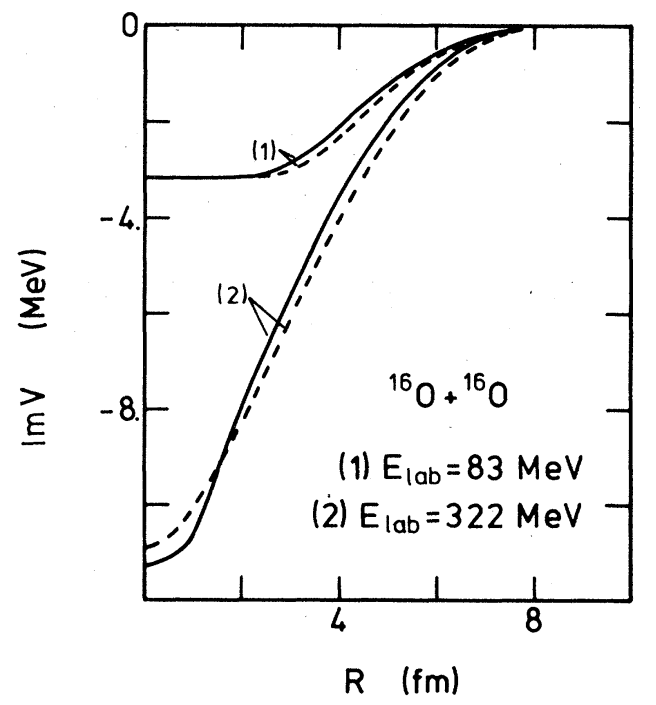

FIG. 4. Comparison of the imaginary part of the optical potential calculated in the present approach (full curve) with that of Ref. 3 based on the finite range complex force $v^{S T}(\overrightarrow{\mathrm{r}})$ (dashed curves) at two laboratory energies. 


$$
E_{\text {pot }}(R, k)=\int H_{\text {pot }}^{(c)}\left[\rho(\overrightarrow{\mathrm{r}}) \tau^{(2)}(\overrightarrow{\mathrm{r}})\right] d \overrightarrow{\mathrm{r}} .
$$

In Eq. (1), the term $E(\infty, k)$ contributes only to the real part.

Using Eqs. (1) and (12) we have calculated the optical potential for ${ }^{16} \mathrm{O}+{ }^{16} \mathrm{O}$ at 83 and $332 \mathrm{MeV}$ laboratory energy. In Fig. 4, we compare our calculations (full curve) for the imaginary part with those of Ref. 3 (dashed curves) obtained from the exact effective interaction. We notice a very close agreement between the two results.

The curves in Fig. 4 correspond to the set SII (Ref. 8) of the Skyrme interaction parameters and $b=1.76 \mathrm{fm}$ for the oscillator parameter. This choice was made by analogy with the calculations for the real part. ${ }^{5}$ The set SIII of Ref. 17 gives almost identical results. The only difference appears at $r<1 \mathrm{fm}$ for $E_{\text {lab }}=332 \mathrm{MeV}$, where $\operatorname{Im} V$ is about $4 \%$ lower for SIII.
These calculations suggest that the parametrization we have proposed for the finite range complex effective interaction appropriately derived for heavy ions ${ }^{3,4}$ is reliable. It can conveniently be used in the derivation of the nucleus-nucleus optical potential as a simpler alternative of the the highly computer-time-consuming approach based on the exact interaction. At present, we study the possibility of applying the method to heavier pairs.

It would be interesting to have a more microscopical foundation of the complex energy functional (9). A density matrix expansion might provide an explicit dependence of its imaginary part on $\rho$ and $\tau$ in a similar way as was found for the real Skyrme interaction. ${ }^{18}$

One of us (R.S.) thanks the Institut Interuniversitaire des Sciences Nucléaires for financial support.
${ }^{1}$ K. -H. Müller, Z. Phys. A $\underline{295}, 79$ (1980).

${ }^{2}$ T. Izumoto, S. Krewald, and A. Faessler, Nucl. Phys. A341, 319 (1980); A357, 471 (1981).

${ }^{3}$ R. Sartor, A. Faessler, S. B. Khadkikar, and S.

Krewald, Nucl. Phys. A359, 467 (1980).

${ }^{4}$ A. Faessler, T. Izumoto, S. Krewald, and R. Sartor, Nucl. Phys. A359, 509 (1980).

${ }^{5}$ D. M. Brink and Fl. Stancu, Nucl. Phys. A243, 175 (1975).

${ }^{6}$ T. H. R. Skyrme, Philos. Mag. 1, 1043 (1956).

${ }^{7}$ P. J. Siemens, Nucl. Phys. A141, 225 (1970).

${ }^{8}$ D. Vautherin and D. M. Brink, Phys. Rev. C $\underline{5}, 626$ (1972).

9J. P. Vary and C. B. Dover, Phys. Rev. Lett. $\underline{31}, 1510$ (1973).

${ }^{10}$ B. Behera, K. C. Panda, and R. K. Sathpathy, Phys. Rev. C 21, 1883 (1980).

${ }^{11}$ G. F. Bertsch, Les Houches Lectures, Session $X X X$, edited by R. Balian et al. (North-Holland, Amster- dam, 1978); G. F. Bertsch, Phys. Rev. C 15,713 (1977).

${ }^{12}$ E. S. Hernandez and S. A. Moszkowski, Phys. Rev. C 21, 929 (1980).

${ }^{13}$ N. J. DiGiacomo, J. C. Peng, and R. M. de Vries, Phys. Lett. 101B, 383 (1981).

${ }^{14}$ Y. M. Engel, D. M. Brink, K. Goeke, S. J. Krieger, and D. Vautherin, Nucl. Phys. A249, 215 (1975).

${ }^{15}$ T. Fliessbach, Z. Phys. 247, 117 (1971).

${ }^{16}$ D. M. Brink, in Many-Body Description of Nuclear Structure and Reactions, Proceedings of the International School of Physics "Enrico Fermi," Course $X X X V I, 1966$, edited by C. Bloch (Academic, New York, 1967), p. 247.

${ }^{17}$ M. Beiner, H. Flocard, Nguyen Van Giai, and P. Quentin, Nucl. Phys. A238, 29 (1975).

18J. W. Negele and D. Vautherin, Phys. Rev. C $\underline{5}, 1472$ (1972). 